\title{
Long-term flux density variations of pulsars: Theoretical structure functions and comparisons with observations
}

\author{
A. Z. Zhou ${ }^{1,3}$, X. J. Wu ${ }^{1,2,3}$, and A. Esamdin ${ }^{2,1,3}$ \\ 1 Department of Astronomy, Peking University, Beijing 100871, PR China \\ 2 the National Astronomical Observatories, the Chinese Academy of Sciences, Beijing 100012, PR China \\ ${ }^{3}$ CAS-PKU Joint Beijing Astrophysics Center, Beijing 100871, PR China
}

Received 6 June 2002 / Accepted 6 March 2003

\begin{abstract}
By means of the refractive interstellar scintillation theory (RISS), the flux density structure functions of PSRs B1642-03, B0736-40, B0740-28 and B0329+54 are calculated and compared with the observations at $610 \mathrm{MHz}$ by Stinebring et al. (2000, hereafter S2000). The theoretical results are in good agreement with observations and the spectra of the electron density fluctuation are all consistent with the Kolmogorov spectra. The theoretical modulation indices $m$ are comparatively less sensitive to the distance $H$ from the observer to the scattering screen but critically depend on the scattering strength $\overline{C_{N}^{2}}$. The structure function does not change remarkably with the variation of $H$ if the scattering screen is closer to the pulsar than to the observer. The results in this paper indicate that the flux density variations observed for these four pulsars are due to a propagation effect (refractive scintillation), not to the intrinsic variability.
\end{abstract}

Key words. stars: pulsars: general - radio continuum: stars - ISM: structure - scattering

\section{Introduction}

The phenomenon of pulsar scintillation was recognized soon after the discovery of pulsars (Hewish et al. 1968) and this field has grown significantly since then (Cordes et al. 1986; Gupta et al. 1993, 1994; Armstrong et al. 1995).

Radio waves propagating in the interstellar medium are scattered by the galactic electrons with random density fluctuations. The scattering process will enlarge the angular sizes of pulsars and produce random flux density fluctuations in space and frequency domains on the observing plane. The spatial variations are mapped to temporal variations by the relative motion between the pulsar, scintillation medium and the observer.

In the strong scattering region, pulsar scintillation is characterized by two kinds of propagation effects: diffractive and refractive interstellar scintillation (DISS and RISS). DISS, which was recognized soon after the discovery of pulsars, is produced by the scattering of pulsar signals by small spatial scale density fluctuations $\left(10^{6}-10^{8} \mathrm{~m}\right)$ in the ISM. DISS causes flux density variations in both time and frequency with characteristic scales of minutes to hours and of $100 \mathrm{kHz}$ to $1 \mathrm{MHz}$, respectively. The long-term flux density variations correlated with DM with the characteristic timescale of days to months is caused by the RISS effects (Sieber 1982; Rickett et al. 1984). RISS is believed to arise from propagation through large-scale

Send offprint requests to: A. Z. Zhou, e-mail: bzaz@bac.pku.edu.cn $\left(10^{10}-10^{12} \mathrm{~m}\right)$ inhomogeneities of electron density in the interstellar medium and hence is a powerful tool to probe the ISM at such scales (Rickett 1990). No consensus has yet been reached on the exact nature of the inhomogeneities of the interstellar medium that result in the refractive effects.

There has been considerable progress over recent years in our understanding of refractive scintillation - both at theoretical and observational levels. On the theoretical front, some people studied the properties of the ISM and the spectrum of density inhomogeneities. Bhat et al. (1998) suggested that the distribution of scattering material in the local ISM is not uniform and proposed the presence of the local bubble. Bhat et al. (1999b) studied the modulations of scintillation observables, flux density and drifting bands in dynamic spectrum. They discussed many different models proposed for the electron density fluctuation spectrum and constrained the electron density spectrum in the local ISM. Bhat \& Gupta (2002) took a more detailed look at the distribution of scattering material toward Loop I and beyond. On the observational front, significant work has been done on the flux density monitoring (Rickett et al. 1990; LaBrecque et al. 1994; Esamdin et al. 2000) and the short timescale variations in dynamic spectra (Gupta et al. 1994; Stinebring et al. 1996; Wang et al. 2001). It is concluded that the diffractive and refractive scintillation are caused by the same underlying scattering process. Furthermore, the measurement of DISS parameters (Bhat et al. 1999a) is used to derive the scattering strength and scintillation speeds, and Bhat et al. (1999a) show that scintillation speeds are reasonably 
good indicators of proper motion speeds. Recently, Stinebring et al. (2000, hereafter S2000) monitored the radio flux density of 21 pulsars at $610 \mathrm{MHZ}$ for five years and presented the structure functions of flux density time series.

Descriptions in terms of structure functions of observable quantities are extensively used to analyze the flux density time series. Romani et al. (1986) predicted the cross-correlation properties of decorrelation bandwidth, scintillation timescale and the flux. Many authors' work attempts to examine the relevant theoretical predictions. Observations of PSR B0329+54 by Stinebring et al. (1996) show that the correlation properties between variations of flux, decorrelation bandwidth and scintillation time are in agreement with the theoretical predictions. Bhat et al. (1999c) analyzed the cross-correlation of the fluctuations of various parameters. The reasonable agreement between the predicted and the measured correlations for some pulsars strongly confirms RISS as the primary cause of the observed fluctuations. Bhat et al. (1999c) also suggest that more comprehensive theoretical investigations to describe observables and their cross-correlations are needed.

However, other authors (Stinebring \& Condon 1990; Kaspi $\&$ Stinebring 1992) have pointed out that the observed variation of pulsar flux densities at radio frequencies can be attributed to either intrinsic luminosity fluctuations or propagation effects, or to some combination of both. Wu et al. (1995) suggested that the variations of the pulse flux density may be one of the important causes of DM fluctuations. Calculating the structure functions by means of RISS theory and comparing with the observations is a method to distinguish propagation effects from intrinsic variations. Although Qian (1995) semiquantitatively calculated the flux density structure functions of PSR B2217+47 in multiple frequencies: 0.31, 0.42, 0.61 and $0.75 \mathrm{GHz}$, the quantitative theoretical calculations have not been thoroughly carried out in the previous work. Therefore, the purpose of this paper is to investigate the flux density structure functions of the four pulsars and compare them with observations and furthermore to find some clues to distinguish refractive interstellar scintillation from intrinsic variations. We have also studied the influence of the variation of the parameters (the scintillation velocity, the scattering strength, etc.) on the structure functions. The paper is organized as follows. In Sect. 2, we describe the RISS theory in brief, and in Sect. 3 the adoption of the parameters are analyzed. In Sect. 4, the theoretical results and their comparison with observations are presented. Finally, we summarize our conclusions and discussion in Sect. 5.

\section{Brief description of RISS theory}

Ramani et al. (1986) and Blandford et al. (1986) have proposed the Riss theory, which can be used to calculate the structure functions of the normalized flux fluctuations of pulsars through the interstellar medium. Blandford et al. (1986) assume that the scattering density distribution of the extended medium is a Gaussian:

$$
\frac{\mathrm{d} Q_{0}}{\mathrm{~d} z}=\frac{2 Q_{*}}{\sqrt{\pi} H} \exp \left(-\frac{L^{2}}{H^{2}}\right)
$$

where $Q_{*}$ is the total scattering strength of the extended medium, $H$ is the $1 / \mathrm{e}$ width of the distribution and $L$ is the distance to the observer.

It is also assumed that the spectrum of the electron density fluctuations follows the isotropic power-law:

$p(\kappa)=C_{N}^{2}(z) \kappa^{-\beta}$ for $\kappa_{\text {out }} \leq \kappa \leq \kappa_{\mathrm{inn}}$,

where $\kappa=2 \pi / s$ is the spatial wave number of the fluctuation, $s$ is the spatial scale, $\kappa_{\text {inn }}$ and $\kappa_{\text {out }}$ are respectively the inner and outer cutoffs of scale size. The indicator of the rms of electron density fluctuations, $C_{N}^{2}$, characterizes the strength of the scattering and is a function of location $(z)$ in the Galaxy. The value of the power-law index $\beta$, which is in the range $3<\beta<5$, determines whether the scattering is thought of as a turbulence spectrum $(\beta<4)$ or a stochastic superposition of discrete scattering structures $(\beta>4)$. Kolmogorov turbulence of the index $\beta=11 / 3$, is an often-discussed model in the literature.

The power spectrum of the phase fluctuations of the wavefronts is also the power-law:

$P(\kappa)=\frac{\lambda^{2}}{(2 \pi)^{4}} Q_{*} \kappa^{-\beta}$.

The autocorrelation function $F(\tau)$ and the structure function $D(\tau)$ can be written as:

$$
\begin{aligned}
F(\tau)=< & \delta F(t) \delta F(t+\tau)>_{t} \\
D(\tau)= & 2 \times[F(0)-F(\tau)]=2 \times\left[<\delta F(t) \delta F(t)>_{t}\right. \\
& \left.-<\delta F(t) \delta F(t+\tau)>_{t}\right]
\end{aligned}
$$

where $\delta F(t)$ represents the normalized flux density fluctuation, $\tau$ is the time lag and the subscript $t$ means averaging over all $t$.

The autocorrelation function can be deduced from RISS theory as (Qian 1995):

$$
\begin{aligned}
F(\tau)= & \frac{\left(\frac{\lambda}{2 \pi}\right)^{4} \Gamma(3-\beta / 2)}{2^{\beta / 2-1} \pi D^{2}} \int_{0}^{D} \mathrm{~d} z(D-z)^{2} z^{2} \frac{\mathrm{d} Q_{0}}{\mathrm{~d} z} \delta^{\beta-6} \\
& M\left(\frac{6-\beta}{2}, 1,-\frac{V^{2} \tau^{2}}{2 \delta^{2}}\left(\frac{z}{D}\right)^{2}\right)
\end{aligned}
$$

where $M$ is the confluent hypergeometric function, $D$ is the distance of the source, $V$ is the transverse velocity of the source relative to the observer, $\Gamma$ is the Gamma function, $\lambda$ is the observation wavelength, $\delta(z)=L^{*} \theta_{\mathrm{s}}$, and $\theta_{\mathrm{s}}$ is the apparent angular radius of the source at the distance $L$.

For $\beta=11 / 3$ (Kolmogorov spectrum), the autocorrelation function is (Qian 1995):

$$
\begin{aligned}
F(\tau)= & \frac{2 Q_{*}\left(\frac{\lambda}{2 \pi}\right)^{4} \Gamma(7 / 6)}{\pi^{3 / 2} 2^{5 / 6}} H^{-1 / 3} \int_{0}^{d_{H}} \mathrm{~d} y y^{-1 / 3} \\
& \left\langle\theta_{\mathrm{s}}^{2}(y)\right\rangle^{-7 / 6}\left(1-\frac{y}{d_{H}}\right)^{2} \mathrm{e}^{-y^{2}} M(7 / 6,1,-x),
\end{aligned}
$$

$x=\frac{V^{2} \tau^{2}\left(1-\frac{y}{d_{H}}\right)^{2}}{2 H^{2} y^{2} \theta_{\mathrm{s}}^{2}(y)}, d_{H}=\frac{D}{H}, y=\frac{L}{H}$, 
and for $\beta=4$,

$$
\begin{aligned}
& F(\tau)=\frac{Q_{*}\left(\frac{\lambda}{2 \pi}\right)^{4}}{\pi^{3 / 2}} \int_{0}^{d_{H}} \mathrm{~d} y\left\langle\Theta_{\mathrm{s}}^{2}(y)\right\rangle^{-1}\left(1-\frac{y}{d_{H}}\right)^{2} \\
& \quad \times \mathrm{e}^{-y^{2}} M(1,1,-x), \\
& x=\frac{V^{2} \tau^{2}\left(1-\frac{y}{d_{H}}\right)^{2}}{2 H^{2} y^{2} \Theta_{\mathrm{s}}^{2}(y)}, d_{H}=\frac{D}{H}, y=\frac{L}{H} .
\end{aligned}
$$

Both the total scattering strength $Q_{*}$ and the scattering angular radius $\theta_{\mathrm{s}}$ depend on the spectrum index and could be obtained as (Romani et al. 1986):

(1) $\beta=11 / 3$

$Q_{*}=3.7 \times 10^{-18} \mathrm{DC}_{-4}\left(\mathrm{~cm}^{-11 / 3}\right)$,

$\theta_{\mathrm{s}}=2.2 D^{0.6} C_{-4}^{0.6} \lambda^{2.2}$ (mas),

(2) $\beta=4$

$Q_{*}=1.6 \times 10^{-21} D C_{-4}\left(\mathrm{~cm}^{-4}\right)$,

$\theta_{\mathrm{s}}=2.2 D^{0.5} C_{-4}^{0.6} \lambda^{2}$ (mas), where $D$ and $\lambda$ are, respectively, in units of kpc and $\mathrm{m}$ and $C_{-4}=\overline{C_{N}^{2}} / 10^{-4}$.

\section{Adoption of the parameters}

Stinebring et al. (2000) plotted the structure functions of the unsmoothed flux density time series for 21 pulsars, of which two pulsars' flux density structure functions do not have wellcharacterized slopes and that of thirteen pulsars have a single slope. However, for the remaining six pulsars, there is a change in the slope of the flux density structure function, corresponding to a change in the slope of the electron density fluctuation spectrum at a spatial scale of about $10^{13} \mathrm{~cm}$. In this paper, the flux density structure functions of four pulsars, PSRs B1642-03, B0329+54, B0736-40 and B0740-28, have been selected to study because each of them has a apparent saturation region and a single slope.

We use the Eqs. (7)-(10) of the RISS model given above to calculate the structure function $D(\tau)$, which have five parameters: $\beta, D, V, H$ and $C_{-4}$.

The pulsar distance can be obtained from the association with supernova remnants, interferometric measurements of annual parallax, H I absorption measurements and dispersion measurements. Lyne et al. (1985) concluded that the typical errors of distance estimates were generally factors of 1.5-2. Now the electron density model of Taylor \& Cordes (1993, hereafter TC93) has been widely used to obtain the pulsar distance from DM measurements, which gives errors of about $25 \%$ for most pulsars. In the latest electron density model, Cordes \& Lazio (2002, hereafter NE2001) made a few important revisions of TC93 model. In this paper, the pulsar distances are taken from S2000, where the distances of PSR B0329+54 and PSR B0740-28 are derived from DM measurements from the TC93 model, while for the other two pulsars, PSR B164203 and PSR B0736-40, the distances are available from H I absorption measurements (Frail \& Weisberg 1990, hereafter FW90) and the distance errors could be better or worse than $25 \%$, depending on the quality of the data. The distances adopted above for the four pulsars are closer to those from NE2001.
The velocity is also an important observational parameter. There are three main methods to estimate the space velocities of radio pulsars. Pulsar proper motion velocity can be obtained from timing and the interferometric measurements (e.g. Harrison et al. 1993). The transverse speed can also be estimated from the interstellar scintillation measurement. The scintillation velocity represents the transverse velocity relative to the Earth and the scattering screen, namely $\boldsymbol{V}_{\mathrm{ISS}}=\boldsymbol{V}_{\mathrm{pm}}+\boldsymbol{V}_{\mathrm{s}}+\boldsymbol{V}_{\mathrm{E}}$, where $\boldsymbol{V}_{\mathrm{pm}}, \boldsymbol{V}_{\mathrm{s}}$ and $\boldsymbol{V}_{\mathrm{E}}$ are the proper motion velocity, the velocity of the scattering screen and that of the Earth respectively. Statistically, the earth motion causes a bias in the scintillation speed that is about $30 \mathrm{~km} \mathrm{~s}^{-1}$, and the bulk flow of the density irregularities in the ISM is less than $10 \mathrm{~km} \mathrm{~s}^{-1}$ (Gupta et al. 1999a). The $\boldsymbol{V}_{\text {ISS }}$ is estimated as $A_{V} \times 10^{4} \frac{\sqrt{\Delta v_{\mathrm{d}} D_{\mathrm{kpc}} x}}{v \tau_{\mathrm{d}}}$, where $\boldsymbol{V}_{\mathrm{ISS}}$ is in $\mathrm{km} \mathrm{s}^{-1}, \Delta v_{\mathrm{d}}$ in MHz, $D$ in kpc, $v$ in GHz and $\tau_{\mathrm{d}}$ in seconds, $x=\frac{D_{0}}{D_{\mathrm{p}}}, D_{0}$ and $D_{\mathrm{p}}$ are the distance from the observer to the screen and that from the screen to the pulsar (for a screen placed midway between the pulsar and the observer, $x=1)$. The constant $A_{V}$ was taken to be $3.85 \times 10^{4}$ by Gupta et al. (1994) and refined to be $2.53 \times 10^{4}$ by Cordes \& Rickett (1998). It is known that there is a good agreement between the scintillation velocity and the proper motion velocity in terms of the scattering screen locating midway between the observer and the pulsar (Gupta 1995), therefore scintillation speeds are generally considered to be useful indicators of a pulsar's proper motion speed (Bhat et al. 1999a).

The power-law index, $\beta$, reflects the fundamental physical information of the scattering medium. The Kolmogorov turbulence $(\beta=11 / 3)$ is produced by a cascade of wave energy from the outer scale to the inner scale cut-off; and the Super-Kolmogorov spectrum with the steeper power-law index $(\beta=4)$ results from the random superposition of discontinuities of the medium, such as a collection of the shock fronts (Rickett 1990). Both the case of $\beta=11 / 3$ and $\beta=4$ are adopted to calculate the flux density structure functions for all the four pulsars.

In our calculation, the distance $(D)$ and the velocity $(V)$ are two physical parameters, while the other three parameters $\left(\beta, C_{-4}\right.$ and $H$ ) are considered to be free. In this paper, $\beta=11 / 3$ and $\beta=4$ both have been taken for calculation for each pulsar.

\section{Theoretical results for four pulsars}

The theoretically calculated structure functions (the solid lines) and their comparison with the observational data of S2000 (the solid dots) are presented in Figs. 1-4.

There are three important quantities of the structure functions to characterize the properties of pulsar flux density variation: $m, \tau_{\mathrm{r}}$ and $p$. The modulation index $m$ denotes the amplitude of the flux density fluctuations and is defined as $m=$ $\sqrt{D_{\infty} / 2}$, where $D_{\infty}$ is the saturation value. $\tau_{\mathrm{r}}$ is the value of the scintillation timescale, the time lag at which the structure function reaches half its saturation value. $p$ is the logarithmic slope of the structure function, which is related to the spectrum of electron density fluctuations in ISM and the distribution along the line of sight. The relation of $D(\tau) \propto \tau^{p}$ is used to calculate $p$. 
Table 1. The best parameter values for theoretical results and the comparison with some observational ones from S2000.

\begin{tabular}{cccccccc}
\hline \hline PSR B & $\beta$ & $\begin{array}{c}D \\
(\mathrm{kpc})\end{array}$ & $\begin{array}{c}H \\
(\mathrm{kpc})\end{array}$ & $\begin{array}{c}V_{\mathrm{t}} \\
\left(\mathrm{km} \mathrm{s}^{-1}\right)\end{array}$ & $\begin{array}{c}V_{\mathrm{pm}} \\
\left(\mathrm{km} \mathrm{s}^{-1}\right)\end{array}$ & $\begin{array}{c}C_{-4, \mathrm{t}} \\
\left(\mathrm{m}^{-20 / 3}\right)\end{array}$ & $\begin{array}{c}C_{-4, \mathrm{DISS}} \\
\left(\mathrm{m}^{-20 / 3}\right)\end{array}$ \\
\hline $1642-03$ & $11 / 3$ & 0.5 & 0.25 & 110 & 110 & 1.8 & 20 \\
$0329+54$ & $11 / 3$ & 1.4 & 0.7 & 83 & 140 & 0.8 & 4 \\
$0736-40$ & $11 / 3$ & 2.1 & 1.05 & 770 & 770 & $10^{4.1}$ & $10^{4.1}$ \\
$0740-28$ & $11 / 3$ & 1.9 & 0.95 & 240 & 240 & 100 & 100 \\
\hline
\end{tabular}
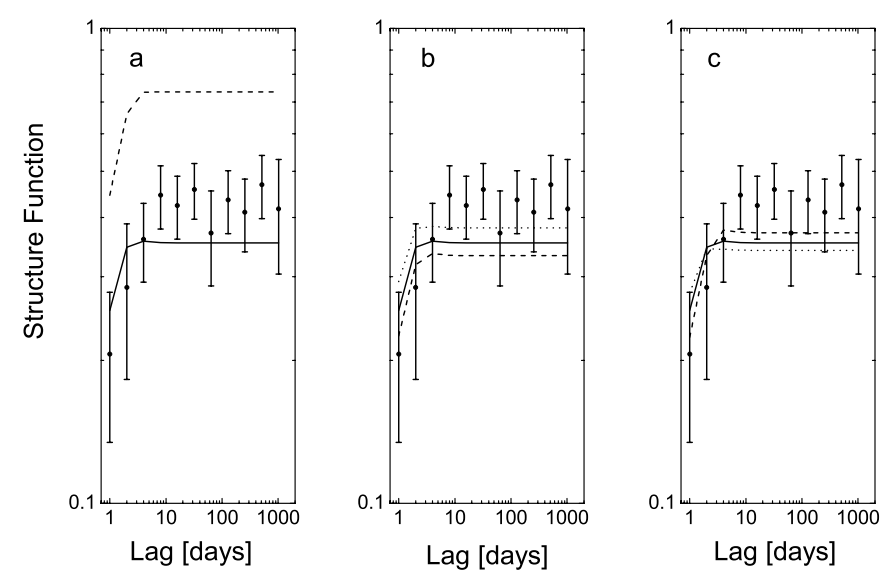

Fig. 1. The theoretical structure functions and the comparison with observations for PSR B1642-03. a) The case for the best fit parameters: the solid and dashed lines represent the structure functions for $\beta=11 / 3$ and $\beta=4$, respectively. b) The case where $C_{-4}$ is treated as free parameter: the dashed, solid and dotted lines represent $C_{-4}=2.1,1.8$ and $1.5 \mathrm{~m}^{-20 / 3}$, respectively. c) The case where $H$ is treated as free parameter: the dashed, solid and dotted lines represent $H=0.4,0.25$ and $0.2 \mathrm{kpc}$, respectively. (Note: Solid dots are observational data from S2000. Solid lines represent theoretical results by the adoption of the best parameters listed in Table 1. Dashed and dotted lines show the influence of the variation of one parameter on the structure function while the other four parameters kept constant from Table 1.)

\subsection{PSR B1642-03}

It is practical to obtain a set of the reasonable parameters in terms of the treatments above, which will be described in detail with respect to the pulsar PSR B1642-03.

The pulsar distance $0.5 \mathrm{kpc}$ is derived from the $\mathrm{H}$ I absorption measurements (FW 90) and the proper motion velocity $110 \mathrm{~km} \mathrm{~s}^{-1}$ is given by Lyne et al. (1982). For the Kolmogorov spectrum, the value $C_{-4}$ can be determined due to the relatively independent effects of $\mathrm{C}_{-4}$ and $H$ on the structure function. Such a property is shown clearly in Figs. $1 \mathrm{~b}$ and $1 \mathrm{c}$ i.e., the saturation value (viz. modulation index) is predominantly controlled by the value $C_{-4}$, while not influenced by the value of $H$, thereby the observed modulation index within the error domain can give a strict restriction on the possible range of $C_{-4}$ and the best fitted value $1.8 \mathrm{~m}^{-20 / 3}$ is obtained. Then, the timescale depends only on the value of $H$, and a value of $H$ between $0.25 \mathrm{kpc}$ and $0.4 \mathrm{kpc}$ can lead to theoretical curves being consistent with observations (Fig. 1c). As Bhat et al. (1999a) mentioned, the scattering screen is generally placed
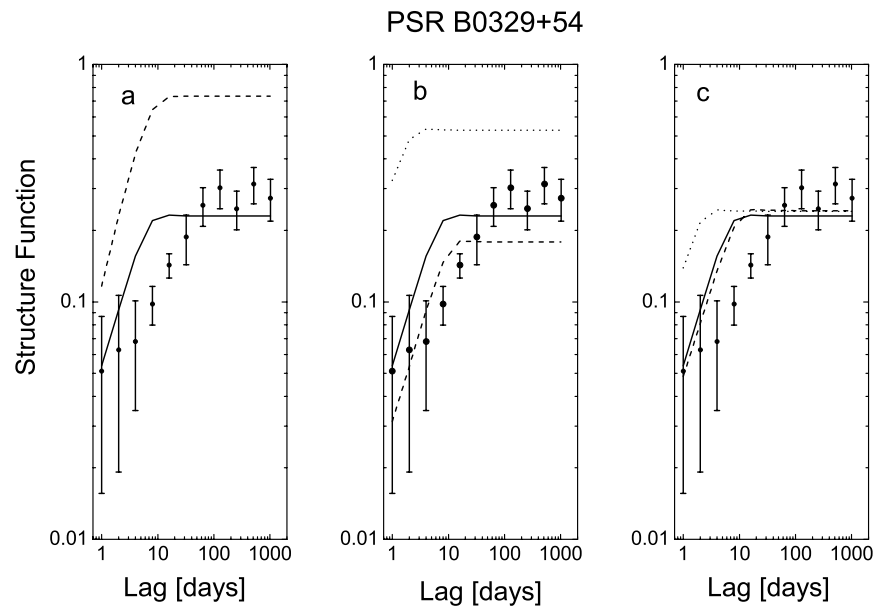

Fig. 2. The theoretical structure functions and the comparison with observations for PSR B0329+54. a) The case for the best fit parameters: the solid and dashed lines represent the structure functions for $\beta=11 / 3$ and $\beta=4$, respectively. b) The case where $C_{-4}$ is treated as free parameter: The dashed, solid and dotted lines represent $C_{-4}=1.5,0.8$ and $0.1 \mathrm{~m}^{-20 / 3}$, respectively. c) The case where $H$ is treated as free parameter: the dashed, solid and dotted lines represent $H=1.2,0.7$ and $0.2 \mathrm{kpc}$, respectively. See the note of caption to Fig. 1 for details.

midway between the observer and the pulsar, therefore we adopt $H=0.25 \mathrm{kpc}$.

However, for the Super-Kolmogorov spectrum, the theoretical curve will always be deviated from the observation no matter how the other four parameters are adjusted. Thus we can conclude that along the line of sight to this pulsar, the spectrum of the electron density fluctuations corresponds to the Kolmogorov form, but not the Super-Kolmogorov form, which is different from $\mathrm{S} 2000$.

In this way, the theoretical structure function curve is obtained (the solid lines in Fig. 1) and a reasonable set of parameters that best match the observational data is also determined as: $D=0.5 \mathrm{kpc}, V=110 \mathrm{~km} \mathrm{~s}^{-1}, \beta=11 / 3, C_{-4}=1.8 \mathrm{~m}^{-20 / 3}$, $H=0.25 \mathrm{kpc}$.

Considering $D$ and $V$ as constant, in order to analyze how the flux density structure functions are influenced by the variations of the three free parameters $\beta, C_{-4}$ and $H$, each time we make one of them variable and keep the other two constant in each panel of Fig. 1.

Figure $1 \mathrm{~b}$ displays the influence of $\mathrm{C}_{-4}$ on the structure function. $C_{-4}=1.5,1.8$ and $2.1 \mathrm{~m}^{-20 / 3}$ are chosen for calculation and the structure function curve will move parallel and downwards with $\mathrm{C}_{-4}$ increasing, which suggests that the 
PSR B0736-40

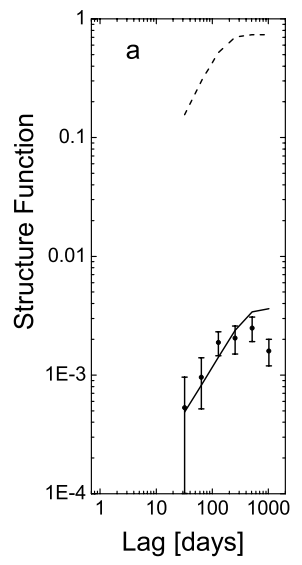

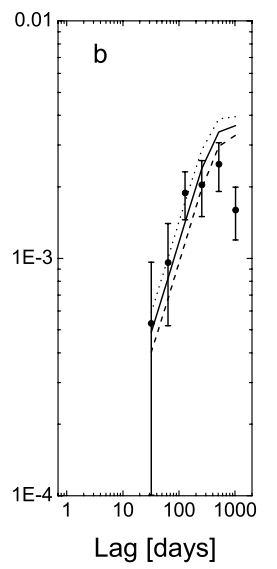

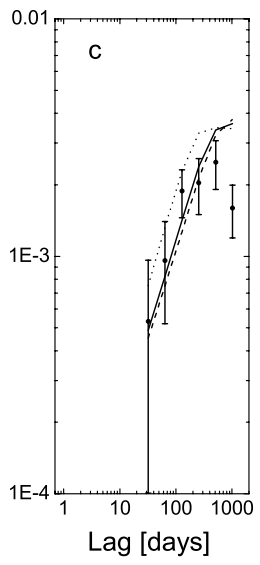

Lag [days]

Fig. 3. The theoretical structure functions and the comparison with observations for PSR B0736-40. a) The case for best fit parameters: the solid and dashed lines represent the structure functions for $\beta=11 / 3$ and $\beta=4$, respectively. b) The case where $C_{-4}$ is treated as free parameter: the dashed, solid and dotted lines represent $C_{-4}=10^{4.2}, 10^{4.1}$ and $10^{4} \mathrm{~m}^{-20 / 3}$, respectively. c) The case where $H$ is treated as free parameter: the dashed, solid and dotted lines represent $H=1.5,1.05$ and $0.5 \mathrm{kpc}$ respectively. See the note of caption to Fig. 1 for details.

logarithmic slope of the structure function is insensitive to the parameter $C_{-4}$. Moreover, the modulation index will become smaller and the timescale will be longer if $\mathrm{C}_{-4}$ increases. The best fitted value $1.8 \mathrm{~m}^{-20 / 3}$ for $C_{-4}$ is much smaller than the observational value $20 \mathrm{~m}^{-20 / 3}$. Such a discrepancy, also seen in pulsar PSR B0329+54, can be interpreted by three possibilities. First, it is partially because of measurement uncertainties in scattering strength. Second, part of this discrepancy is to do with the fact that the adopted distance may be significantly offset from the real value. The third likely explanation is that the observational values of scattering strength are obtained from the dynamic spectrum due to DISS, while the fitted values are obtained by calculating the structure function with the RISS theory. It may suggest that the interstellar mediums causing DISS and RISS respectively probably lie in different regions and have different spatial scales that possibly bring different scattering strength.

For the sensitivity of the structure function to the value of $H$, Fig. 1c shows that the structure function does not change remarkably with the variation of $H$ if $H>D / 2$. However, when $H<D / 2$, the timescale will become slightly shorter with $H$ decreasing, while $m$ and $p$ will not change.

Furthermore, we find that the flux density structure functions of the other three pulsars are under a similar influence of the variations of the free parameters, therefore a similar calculation procedure has been carried out for each of them for the Kolmogorov spectrum.

The best sets of five parameter values used to calculate the theoretical flux density structure functions and some of the observational ones from $\mathrm{S} 2000$ for comparison are listed in Table 1, where the distances and the proper motion velocities are taken from $\mathrm{S} 2000, V_{\mathrm{t}}$ is the best satisfied velocity in the theoretical calculations and $C_{-4}$ has two values, the theoretical fit

PSR B0740-28
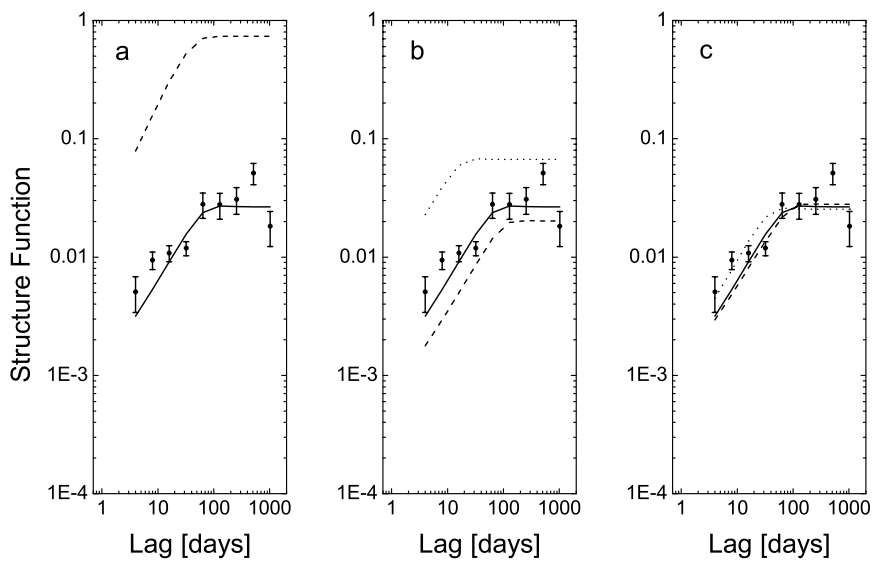

Fig. 4. The theoretical structure functions and the comparison with observations for PSR B0740-28. a) The case for best fit parameters: the solid and dashed lines represent the structure functions for $\beta=11 / 3$ and $\beta=4$, respectively. b) The case where $C_{-4}$ is treated as free parameter: the dashed, solid and dotted lines represent $C_{-4}=200,100$ and $10 \mathrm{~m}^{-20 / 3}$, respectively. c) The case where $H$ is treated as free parameter: the dashed, solid and dotted lines represent $H=1.5,0.95$ and $0.5 \mathrm{kpc}$, respectively. See the note of caption to Fig. 1 for details.

results and the observed values (calculated by $C_{-4}=\overline{C_{N}^{2}} / 10^{-4}$ ) from DISS measurements (S2000). The theoretical and observational values of $m, \tau_{\mathrm{r}}$ and $p$ are listed in Table 2, where subscripts " $t$ " and "o" represent the theoretical and the observational values, respectively.

\subsection{PSR B0329+54}

Along the line of sight to this pulsar, the spectrum of electron density fluctuations is still uncertain. According to S2000, it is either a Kolmogorov or Super-Kolmogorov spectrum. Our calculation is mainly to examine the nature of the electron density fluctuation spectrum.

For $\beta=4$, like in the situation of PSR B1642-03, the theoretical curve will always depart very far from observations no matter how the other parameters are modulated, so the case of $\beta=4$ can be ruled out.

For $\beta=11 / 3$, by comparing the theoretical modulation index with the observational value 0.37 , a value of $0.8 \mathrm{~m}^{-20 / 3}$ for $C_{-4}$ is best fitted, which is smaller than the observational value $4 \mathrm{~m}^{-20 / 3}$. The best fit value $H=0.7 \mathrm{kpc}(D / 2)$ means that the scattering screen is located midway between the observer and the pulsar, which agrees with Bhat et al. (1999a).

The distance $1.4 \mathrm{kpc}$ derived from the TC93 model is taken for calculation. However, as for the velocity, it can be obtained from many different measurements. Gupta (1995) gave the scintillation velocity and the proper motion velocity, $126 \mathrm{~km} \mathrm{~s}^{-1}$ and $145 \mathrm{~km} \mathrm{~s}^{-1}$, respectively. Later on, the proper motions of pulsars were improved by Fomalont et al. (1997) with VLA observations and they figured out the transverse velocity of $135 \pm 35 \mathrm{~km} \mathrm{~s}^{-1}$ according to their measurements. There is not much discrepancy among these velocity values. However, with the observational parameters of S2000 and the 
Table 2. The theoretical and observational values of modulation indices, logarithmic slopes and refractive timescales.

\begin{tabular}{cccccccc}
\hline \hline PSR B & $\beta$ & $m_{\mathrm{o}}$ & $m_{\mathrm{t}}$ & $\begin{array}{c}\tau_{\mathrm{r}, \mathrm{o}} \\
(\text { days })\end{array}$ & $\begin{array}{c}\tau_{\mathrm{r}, \mathrm{t}} \\
(\text { days })\end{array}$ & $p_{\mathrm{o}}$ & $p_{\mathrm{t}}$ \\
\hline $1642-03$ & $11 / 3$ & $0.46 \pm 0.04$ & 0.42 & 0.9 & 0.6 & 0.4 & 0.76 \\
$0329+54$ & $11 / 3$ & $0.37 \pm 0.03$ & 0.34 & 15 & 4 & 0.5 & 0.78 \\
$0736-40$ & $11 / 3$ & $0.03 \pm 0.006$ & 0.04 & 71 & 128 & 0.7 & 0.77 \\
$0740-28$ & $11 / 3$ & $0.13 \pm 0.01$ & 0.12 & 29 & 26 & 0.5 & 0.76 \\
\hline
\end{tabular}

refined constant $A_{V}$ in the scintillation velocity calculation formula given by Cordes \& Rickett (1998), a smaller scintillation velocity of $83 \mathrm{~km} \mathrm{~s}^{-1}$ is obtained. It is found that either the transverse velocity $135 \mathrm{~km} \mathrm{~s}^{-1}$ or the scintillation velocity $126 \mathrm{~km} \mathrm{~s}^{-1}$ makes the theoretical curve approximate to the observation, but can't result in the allowable theoretical curve within the range of observational error and the timescale is too much smaller than the observational value. However, the adoption of $83 \mathrm{~km} \mathrm{~s}^{-1}$ can give a better match with observations. It may indicate that for this pulsar the scintillation velocity is substantially smaller than the proper motion velocity, as mentioned by Gupta et al. (1994). The likely interpretation is that the bulk flow of the medium and the orbital motion of the Earth also play important roles in determining timescales (Bhat et al. 1998). Figure 2 a shows that the electron density fluctuation spectrum is much closer to the Kolmogorov form than to the Super-Kolmogorov form along the line of sight to this pulsar. We find that the agreement is better for even lower values of velocity.

\subsection{PSR B0736-40 and PSR B0740-28}

The cases of pulsars PSR B0736-40 and PSR B0740-28 are very similar in terms of the adoption of parameters and the theoretical results. For PSR B0736-40, the distance value $2.1 \mathrm{kpc}$ is derived from H I absorption measurements (FW90) and the proper motion velocity $770 \mathrm{~km} \mathrm{~s}^{-1}$ is calculated from proper motion measurements by Fomalont et al. (1992). For PSR B0740-28, the distance value $1.9 \mathrm{kpc}$ is converted from DM using the TC93 model and the proper motion velocity $240 \mathrm{~km} \mathrm{~s}^{-1}$ is from the proper motion measurements by Bailes et al. (1992). For the Kolmogorov spectrum, the theoretical results are in good agreement with observations. The best fitted values of $C_{-4}$ are equal to the observational values of DISS measurements from S2000. The best fitted values of $H$ are $1.05 \mathrm{kpc}$ and $0.95 \mathrm{kpc}$, respectively, showing that the scattering screen is placed midway between the pulsar and the observer, which also agrees with Bhat et al. (1999a).

The theoretical curves for $\beta=4$, however, deviate very far from observations no matter how the parameters are adjusted. Therefore, the spectra of the electron density fluctuations fit the Kolmogorov spectrum, but not the Super-Kolmogorov spectrum. It confirms the conclusion of S2000 with respect to the nature of the electron density fluctuation spectra along the lines of sights to the two pulsars.

The effects of the parameter variations on the structure functions (from Figs. 3 and 4) for these two pulsars are the same as that for pulsars PSR B1642-03 and PSR B0329+54.

\section{Influence of uncertainties of $D$ and $V$ on structure functions}

Although the distance and velocity are regarded as certain values in the calculation above, in order to investigate the influence of their uncertainties on structure functions, we modulate them within the reasonable ranges to obtain the corresponding structure functions.

The distances are made variable within the typical error range at about $25 \%$ to calculate the structure function. Although the modulation indices and the timescales can be slightly changed by the variations of distance, the distance uncertainties generally have no significant influence on structure functions. Therefore, the effects of distance errors on the structure function can be negligible.

Since the maximum error in estimating a pulsar's speed is $\pm 30 \mathrm{~km} \mathrm{~s}^{-1}$ (Cordes 1986), the velocities are modulated in range of $30 \mathrm{~km} \mathrm{~s}^{-1}$ to calculate the structure functions. For PSRs B1642-03, B0736-40 and B0740-28, the intensity structure functions do not change with the velocity variations. The larger uncertainties in the best fitted velocity for PSR B0329+54 may be due to the comparatively larger errors of the structure function values at lower lags.

\section{Conclusions and discussions}

The theoretical flux density structure functions and the most reasonable parameters for these four pulsars are obtained by means of the RISS theory. The theoretical values of modulation indices, logarithmic slopes and the timescales are also calculated and compared with the observational values. From the theoretical results, the electron density fluctuation spectra along the lines of sight to these four pulsars are all consistent with the Kolmogorov spectra. For the Super-Kolmogorov spectrum, the theoretical curves will always deviate very far from observations no matter how the parameters are adjusted. The modulation indices are comparatively less prone to the location of the scattering screen but critically depend on the scattering strength. Therefore, the modulation index is a good indicator of scattering strength and the former will become smaller with an increase of the latter. For all the four pulsars, the scattering screen located midway between the pulsar and the observer is reasonable, which means $H=D / 2$. The measurement uncertainties of distance and velocity generally have no remarkable influence on structure functions. Our results suggest that the long-term flux density variations observed for these four pulsars are due to a propagation effect (refractive scintillation), but not the intrinsic variability. 
Acknowledgements. We wish to thank Professors S. J. Qian and C. M. Zhang for their helpful discussions. We also thank Professor A. G. Lyne for his useful comments and profitable suggestions about our manuscript. This work is supported by the National Natural Science Foundation of China under Grant 10073001.

\section{References}

Armstrong, J. W., Rickett, B. J., \& Spangler, S. R. 1995, ApJ, 443, 209

Bailes, M., Manchester, R. N., Kesteven, M. J., Norris, R. P., \& Reynolds, J. E. 1990, MNRAS, 247, 322

Bhat, N. D. R., Rao, A. P., \& Gupta, Y. 1999a, ApJ, 121, 483

Bhat, N. D. R., Gupta, Y., \& Rao, A. P. 1999b, ApJ, 514, 249

Bhat, N. D. R., Rao, A. P., \& Gupta, Y. 1999c, ApJ, 514, 272

Bhat, N. D. R., Gupta, Y., \& Rao, A. P. 1998, ApJ, 500, 262

Bhat, N. D. R., \& Gupta, Y. 2002, ApJ, 567, 342

Blandford, R., Narayan, R., \& Romani, R. W. 1986, ApJ, 301, L53

Cordes, J. M., \& Lazio, T. J. W. 2002 [astro-ph/0207156] (NE2001)

Cordes, J. M., Piderbetsky, A., \& Lovelace, R. V. E. 1986, ApJ, 310, 737

Cordes, J. M., \& Rickett, B. J. 1998, ApJ, 507, 846

Esamdin, A., Wu, X. J., \& Zhang, J. 2000, ChA\&A, 24, 275

Frail, D. A., \& Weisberg, J. M. 1990, AJ, 100, 743 (FW90)

Fomalont, E. B., Goss, W. M., Lyne, A. G., Manchester, R. N., \& Justtanont, K. 1992, MNRAS, 258, 497
Fomalont, E. B., Goss, W. M., Manchester, R. N., \& Lyne, A. G. 1997, MNRAS, 286, 82

Gupta, Y., Rickett, B. J., \& Coles, W. A. 1993, ApJ, 403, 183

Gupta, Y., Rickett, B. J., \& Lyne, A. G. 1994, MNRAS, 269, 1035

Gupta, Y. 1995, ApJ, 451, 717

Harrison, P. A., Lyne, A. G., \& Anderson, B. 1993, MNRAS, 261, 113

Hewish, A., Bell, S. J., Pilkington, J. D. H., Scott, P. F., \& Collins, R. A. 1968, Nature, 217, 709

Kaspi, V., \& Stinebring, D. 1992, ApJ, 392, 530

LaBrecque, D. R., Rankin, J. M., \& Cordes, J. M. 1994, AJ, 108, 1854

Lyne, A. G., Anderson, B., \& Salter, M. J. 1982, MNRAS, 201, 503

Lyne, A. G., Manchester, R. N., \& Taylor, J. H. 1985, MNRAS, 213, 613

Qian, S. J. 1995, ChA\&A, 19, 523

Rickett, B. J. 1990, ARA\&A, 28, 561

Rickett, B. J., Coles, W. A., \& Bourgois, G. 1984, A\&A, 134, 390

Rickett, B. J., \& Lyne, A. G. 1990, MNRAS, 244, 68

Romani, R. W., Narayan, R., \& Blandford, R. 1986, MNRAS, 220, 19

Sieber, W. 1982, A\&A, 113, 311

Stinebring, D. R., \& Condon, J. J. 1990, ApJ, 352, 207

Stinebring, D. R., Faison, M. D., \& Mckinnon, M. M. 1996, ApJ, 460, 460

Stinebring, D. R., Smirnova, T. V., \& Hankins, T. H., et al. 2000, ApJ, 539,300 (S2000)

Taylor, J. H., \& Cordes, J. M. 1993, ApJ, 411, 674 (TC93)

Wang, N., Wu, X. J., \& Manchester, R. N., et al. 2001, ChJAA, 1, 430

Wu, X. J., \& Chian, A. C. L. 1995, ApJ, 443, 261 